\title{
Perception and Reality of Cognitive Function: Information Processing Speed, Perceived Memory Function, and Perceived Task Difficulty in Older Adults
}

\author{
Anna Torrens-Burton ${ }^{\mathrm{a}}$, Nasreen Basoudan ${ }^{\mathrm{a}}$, Antony J. Bayer ${ }^{\mathrm{b}}$ and Andrea Tales ${ }^{\mathrm{a}, *}$ \\ ${ }^{a}$ Department of Psychology, Swansea University, Singleton Park, Swansea, Wales, UK \\ ${ }^{\mathrm{b}}$ Division of Population Medicine, Cardiff University, University Hospital Llandough, Penarth, Wales, UK
}

Accepted 21 August 2017

\begin{abstract}
This study examines the relationships between two measures of information processing speed associated with executive function (Trail Making Test and a computer-based visual search test), the perceived difficulty of the tasks, and perceived memory function (measured by the Memory Functioning Questionnaire) in older adults (aged 50+y) with normal general health, cognition (Montreal Cognitive Assessment score of 26+), and mood. The participants were recruited from the community rather than through clinical services, and none had ever sought or received help from a health professional for a memory complaint or mental health problem. For both the trail making and the visual search tests, mean information processing speed was not correlated significantly with perceived memory function. Some individuals did, however, reveal substantially slower information processing speeds (outliers) that may have clinical significance and indicate those who may benefit most from further assessment and follow up. For the trail making, but not the visual search task, higher levels of subjective memory dysfunction were associated with a greater perception of task difficulty. The relationship between actual information processing speed and perceived task difficulty also varied with respect to the task used. These findings highlight the importance of taking into account the type of task and metacognition factors when examining the integrity of information processing speed in older adults, particularly as this measure is now specifically cited as a key cognitive subdomain within the diagnostic framework for neurocognitive disorders.
\end{abstract}

Keywords: Aging, information processing speed, metacognition, reaction time, subjective cognitive impairment

\section{INTRODUCTION}

Although debate continues with respect to the theoretical and applied relationship between slowing and cognition, information processing speed is a measure commonly used in research as a behavioral indicator, or proxy, of the integrity of cognitive function. The relationship is underpinned by a substantial body

\footnotetext{
*Correspondence to: Andrea Tales, Department of Psychology, Swansea University, Singleton Park, Swansea, SA2 8PP, Wales, UK. E-mail: A.Tales@swansea.ac.uk.
}

of evidence linking behaviorally measured change in information processing speed to brain structure (e.g., to deterioration in white and grey matter) and function in aging, mild cognitive impairment (MCI), and dementia [1-14]. The potential relevance of information processing speed in research and clinical practice is highlighted by evidence indicating that it can predict ability to perform aspects of daily activity and quality of life [15-20], and it is now specifically cited as a key cognitive subdomain within the diagnostic framework for neurocognitive disorders in DSM-5 [21]. 
Concerns about self-perceived cognitive decline, especially of memory, commonly occur in older adults [22], and it is increasingly apparent that both $\mathrm{MCI}$ and dementia can be characterized by an earlier stage, variously termed subjective cognitive impairment (SCI) or decline (SCD), without any objective evidence of deficit from neuropsychological assessment [22-26]. However, subjective cognitive complaints do not always represent a prodromal stage of dementia, with some causes (e.g., anxiety, depression, and sleep disorder) potentially responsive to intervention [22, 27, 28]. Irrespective of causality, concerns about memory function can impact negatively upon everyday life and mental health, with worry about developing dementia and withdrawal from positive health and social behavior [20, 29].

SCI is characterized by objectively defined normal neuropsychological test performance. It is of course possible that objective change is absent in functions such as memory because the tests used are insensitive or do not measure the specific aspect of memory that an individual perceives as having changed. Detrimental change in brain functions other than memory may occur in what we term SCI, but this may be difficult for the general public to describe and, if not tested, may manifest only as vague perceptions of change. Indeed, emerging evidence indicates that fundamental brain operations may be disrupted in individuals with SCI [30]. Therefore, it is possible that a reduction in integrity of fundamental brain processes may impair memory function to a level that may be perceived by the individual, but not evident from current neuropsychological testing protocols.

Despite evidence of inter-relationships between information processing speed, cognition, white and grey matter integrity and behavior, in aging, MCI and dementia [23, 30, 31], there is a lack of research into information processing speed in relation to $\mathrm{SCI}$, and this is particularly so in those individuals who experience subjective changes in memory function in the absence of formal clinical investigation. It is possible that a slowing of information processing speed in individuals experiencing subjective changes in memory might be indicative of structural change (in white matter for example) to which routine neuropsychological tests are not sensitive, but which affects general brain function, cognition, and the perception of functional integrity. Preservation of information processing speed in individuals reporting impaired memory may indicate structural normality.
In this study, therefore, we examine perceived memory function in relation to information processing speed in community-living older adults who have not approached health care services with concerns about their memory or cognitive function [23, 27] and with normal levels of general cognitive function and no significant anxiety or depression. In addition, as metacognition can be a factor in the self-perception of the integrity of memory and cognition [31], we also ask whether there is any relationship between reported memory performance and the perception of task difficulty (i.e., is high level of perceived memory dysfunction associated with greater perception of task difficulty?) and whether perceived task difficulty is related to actual (objectively measured) speed of information processing.

There is evidence (e.g., [5]) to suggest that the speed of information processing, and thus study outcome, can differ significantly with respect to the test used, because of the different brain networks and processes recruited by specific task demands. We therefore report studies using two different measures, the pen-and-paper-based trail making test (TMT) and a computer-administered visual search task.

The TMT is commonly used in clinical settings and in aging, MCI, and dementia research to examine information processing speed and executive function [32]. Trails A is a one-trial task typically described as probing functions such as speed of processing in relation to attention, visual scanning and search, number recognition, numeric sequencing and motor speed; giving a baseline measure of perceptual processing and motor speed. Trails B is again a one-trial task typically described as probing the efficiency of set-shifting, mental flexibility, executive function, divided attention, attention switching and shifting, visual search set shifting, simultaneous maintenance of two sequences, working memory and cognitive flexibility; and, thus, is arguably a measure of information processing speed in relation to multiple high level, non-specific functions. The computer-based visual search task requires rapid serial information processing and response over numerous trials, with measurement of the time taken to respond to an isolated target (whether an arrow is pointing right or left) and the time taken to respond to the same target when it is surrounded by similar but irrelevant distracters. This allows us to determine information processing speed per se, but also to measure the effect of irrelevant but distracting stimuli upon such processing. 
Table 1

Mean demographic scores and Trails A and Trails B information processing speeds for older adults. Standard deviation in parenthesis. Note that range refers to observed range within the data

\begin{tabular}{lccccccc}
\hline & $\begin{array}{c}\text { Age } \\
(\mathrm{y})\end{array}$ & $\begin{array}{c}\text { Education } \\
(\mathrm{y})\end{array}$ & $\begin{array}{c}\text { MFQ-total } \\
\text { score }\end{array}$ & $\begin{array}{c}\text { Trails A } \\
(\mathrm{s})\end{array}$ & $\begin{array}{c}\text { Perceived } \\
\text { difficulty scale } \\
\text { for Trails A }\end{array}$ & $\begin{array}{c}\text { Trails B } \\
(\mathrm{s})\end{array}$ & $\begin{array}{c}\text { Perceived } \\
\text { difficulty scale } \\
\text { for Trails B }\end{array}$ \\
\hline $\begin{array}{l}\text { Older adults } \\
(n=81)\end{array}$ & $\begin{array}{c}65(5.5) \\
\text { Range 50-79 }\end{array}$ & $16(4.8)$ & $295(49.1)$ & $29.05(9.3)$ & $\begin{array}{c}2(1.2) \\
\text { Range 1-6 }\end{array}$ & $43.43(9.4)$ & $\begin{array}{c}3(1.6) \\
\text { Range 1-6 }\end{array}$ \\
\hline
\end{tabular}

Information processing speed for both Trails A and Trails B is represented by the box plot in Fig. 1. Note the presence of outliers in the performance of this task.

\section{STUDY 1. TRAIL MAKING TEST: METHODS}

This study was approved by the ethics committee of Swansea University, Department of Psychology. All participants gave written informed consent to participate.

\section{Participants}

Community-dwelling older adults $(n=100)$ were recruited through adverts placed in local newspapers and social clubs throughout the Swansea area and by word of mouth. Exclusion criteria included poor selfreported general health, any past history of significant medical, neurological, or mental health problems, evidence of physical slowing (e.g., related to Parkinson's disease or arthritis), or previous visit to a health care professional with memory complaints, anxiety, or depression. From those recruited, 19 individuals were excluded. Of these, 8 had Montreal Cognitive Assessment (MoCA) scores of 24 or 25, 3 had MoCA scores below this; 8 had current or past history of significant medical problems, anxiety, or depression. Of those included in the study $\left(\mathrm{n}^{1}=81\right.$; age $50+\mathrm{y}$; 31 male, 50 female), all had normal or correctedto-normal vision and hearing. Although medication could not be controlled, none of the participants reported receiving medication likely to affect information processing speed or cognitive function. All had normal overall cognition (score of 26 or above) using the MoCA [33] and no significant anxiety or depression, as determined by the Generalized Anxiety Disorder 7-item (GAD-7) score less than 5 $[34,35]$ and the Patient Health Questionnaire (PHQ9) score less than 4 [36], respectively. Participants' age, sex, and years of full time education were recorded. A trained researcher administered the tests.

\footnotetext{
${ }^{1}$ Note that participant estimate number was based on a twotailed analysis with an effect size 0.4 , an alpha of 0.05 , and a power of 0.8: giving an estimate of 46 individuals.
}

Testing took place within the Psychology Department at the University of Swansea.

\section{Subjective memory assessment}

Subjective memory function was measured using the Memory Functioning Questionnaire (MFQ) [37]. This 64-item questionnaire assesses the perception of everyday memory functioning with seven sections on general rating of memory, retrospective functioning (compares current memory with past ability), frequency of forgetting, frequency of forgetting while reading, remembering past events, seriousness of forgetting (how memory impairment impacts daily life), and mnemonics usage. Each item is scored on a 1 to 7 Likert scale $(1=$ severe memory problems; $7=$ no problems). Scores range between 64 and 448 with high scores reflecting less severe memory complaints.

Table 1 shows the demographics and TMT data.

\section{Trail Making Test}

Practice trails were provided for both Trails A and B. For Trails A, the participants were instructed to draw one continuous line joining a series of circled numbers in ascending order on a sheet of paper as fast but as accurately as possible. For Trails B, the participants were instructed to draw one continuous line joining a series of circled numbers and letters alternately in ascending and alphabetical order on a sheet of paper as fast but as accurately as they could. Test outcome was the time taken in seconds to complete the test (with the time required to rectify any error forming part of the information processing speed score). No performance feedback was provided. Immediately after completing each of trails A and $\mathrm{B}$, participants were asked to rate, using a scale of 1 to 7, how easy or difficult they found each test to complete, with 1 very easy to complete and 7 very difficult. Study debrief was performed at the end of the experimental session. 
Table 2

Mean baseline demographics, information processing speed and errors. Standard deviation in parenthesis. Note that range refers to observed range within the data

\begin{tabular}{|c|c|c|c|c|c|c|c|c|}
\hline & \multirow[t]{2}{*}{$\begin{array}{l}\text { Age } \\
(y)\end{array}$} & \multirow[t]{2}{*}{$\begin{array}{c}\text { Education } \\
\text { (y) }\end{array}$} & \multirow[t]{2}{*}{$\begin{array}{l}\text { MFQ Total } \\
\text { score }\end{array}$} & \multicolumn{2}{|c|}{$\begin{array}{c}\text { Information } \\
\text { processing speed }\end{array}$} & \multicolumn{2}{|c|}{ Mean group errors } & \multirow{2}{*}{$\begin{array}{c}\text { Perceived } \\
\text { performance } \\
\text { Likert scale } \\
\end{array}$} \\
\hline & & & & $\begin{array}{l}\text { Target } \\
\text { alone }\end{array}$ & $\begin{array}{l}\text { Target plus } \\
\text { distracters }\end{array}$ & $\begin{array}{l}\text { Target } \\
\text { alone }\end{array}$ & $\begin{array}{l}\text { Target plus } \\
\text { distracters }\end{array}$ & \\
\hline $\begin{array}{l}\text { Older adults } \\
(n=54)\end{array}$ & $\begin{array}{c}66 \\
\text { Range 55-79 } \\
(5.2)\end{array}$ & $15(3.7)$ & $290(46.5)$ & $743.02(164.91)$ & $1685.55(314.23)$ & 0.37 & 0.33 & $\begin{array}{c}3(1.4) \\
\text { Range 1-6 }\end{array}$ \\
\hline
\end{tabular}

Information processing speed for both the Target alone and Target plus distracter conditions is represented by the box plot in Fig. 3 . Note the presence of outliers in the performance of this task.

\section{RESULTS}

\section{TMT: Information processing speed and} subjective memory function

Spearman's correlational analysis showed no association between MFQ total score and information processing speed for either Trails A or B (all $p$ values $>0.05$ ). Of the seven subscales of the MFQ, none showed an association with either Trails A or Trails B (all $p$ values $>0.05$ ) except for a significant negative correlation of Trails A with the Mnemonics subscale $(\mathrm{r}=-0.295, p=0.007$, which survives Bonferroni correction, $p=0.042$ ).

\section{TMT: Information processing speed and perceived task difficulty}

The mean (sd) perceived task difficulty score was 2 (1.2) with a range from 1 to 6 for Trails A and 3 (1.6) with a range of 1 to 6 for Trails B. This was not significantly correlated with performance on Trails A $(p>0.05)$ but was significantly positively correlated with performance on Trails B $(r=0.293$, $p=0.008$ ), with slower actual information processing speed associated with a greater perceived task difficulty. Post hoc tests revealed that these results did not vary with respect to educational level or whether the participant was male or female.

\section{Subjective memory function and perceived task difficulty}

MFQ total score was significantly negatively correlated with perceived task difficulty for Trails A $(\mathrm{r}=-0.275, p=0.013)$ and Trails $\mathrm{B}(\mathrm{r}=-0.334$, $p=0.002$ ), with higher levels of subjective memory complaint related to greater perception of task difficulty. Post hoc tests revealed that these results did not vary with respect to educational level or whether the participant was male or female.

\section{STUDY 2: VISUAL SEARCH TASK: METHODS}

\section{Participants}

In the second study, another (separate) group of older adults $(n=62)$ were recruited. The protocol (i.e., inclusion and exclusion factors) was exactly the same as in study 1, as was the recruitment procedure. From those recruited, 6 individuals were excluded due to MOCA scores of 25 or less, with 2 further individuals excluded as a result of current poor medical health. Demographic details for the participants $\left(n^{2}=54\right.$; age 50+ y; 24 male, 30 female $)$ of this second study are shown in Table 2. All participants completed all 64 items on the MFQ.

\section{Visual Search Task: Experimental task and procedure}

For the computer-based visual search task, the time taken to respond to a target (target discrimination) when it appeared in isolation upon the screen and the time taken to respond to the same target when it was surrounded by similar but irrelevant and distracting stimuli were determined. This paradigm was presented on a Dell Precision PC running on Windows XP X86 CPU, viewed at a distance of $57 \mathrm{~cm}$. All trials included a black target that was either a left- or right-pointing arrow, the task being to indicate whether the arrow was pointing to the right or left. The distracting stimuli consisted of seven black arrows that pointed up or down. A clock-face configuration (see Fig. 2) was used to position the target, both when it appeared alone and when surrounded by 7 distracters, in a counterbalanced arrangement

\footnotetext{
${ }^{2}$ Note that participant estimate number was based on a twotailed analysis with an effect size 0.4 , an alpha of 0.05 , and a power of 0.8: giving an estimate of 46 individuals.
} 
in order to eliminate any differences in processing between right and left and upper and lower visual fields. A total of 64 trials were presented; the target appearing 8 times at each of the possible 'clock-face' locations. For half of the trials distracters were presented at the other locations and for the other half no distracters were presented. For each trial the central fixation cross appeared on screen for $1000 \mathrm{~ms}$ prior to the appearance of the target (with or without distracters) and remained on screen for the duration of the trial. The stimuli remained on screen until the participant responded, after which the fixation point re-appeared. The participants were instructed to fixate on the center cross at the beginning of each trial and to respond as quickly but as accurately as possible to whether the target was pointing to the right or left by pressing one of two computer keyboard keys. After instruction, all participants were asked to reiterate the instructions to ensure understanding and then performed a practice block of no more than 10 trials. The ability of the participants to fixate on the cross at the beginning of each trial continued to be checked throughout the procedure by researcher observation. No performance feedback was provided.

Group mean errors were calculated. Responses were eliminated if they were incorrect or obviously due to a disturbance/lapse of concentration or below $150 \mathrm{~ms}$ (faster than 'natural' reaction time therefore representing the pre-empting of the stimulus). No participants failed to respond to a trial. For each participant, the median time (information processing speed) taken to respond for the target alone and the target plus distracter trials was determined and group mean data produced (see Table 2).

\section{Perception of task difficulty}

Immediately after completing the test participants were asked to rate, using a Likert scale of 1 to 7 , how easy or difficult they found each test to complete, with 1 very easy to complete and 7 very difficult. Study debrief was performed at the end of the experimental session.

\section{RESULTS}

Visual search: information processing speed and subjective memory function

MFQ total score and subscales scores were not significantly correlated with information processing speed for either the target alone or the target plus distracters conditions ( $p$ values $>0.05$ ). Overall errors on the visual search tasks were very small (mean group errors 0.37 for target alone and 0.33 for target plus distracters) and the number of errors was not significantly correlated with MFQ scores ( $p$ values $>0.05$ ).

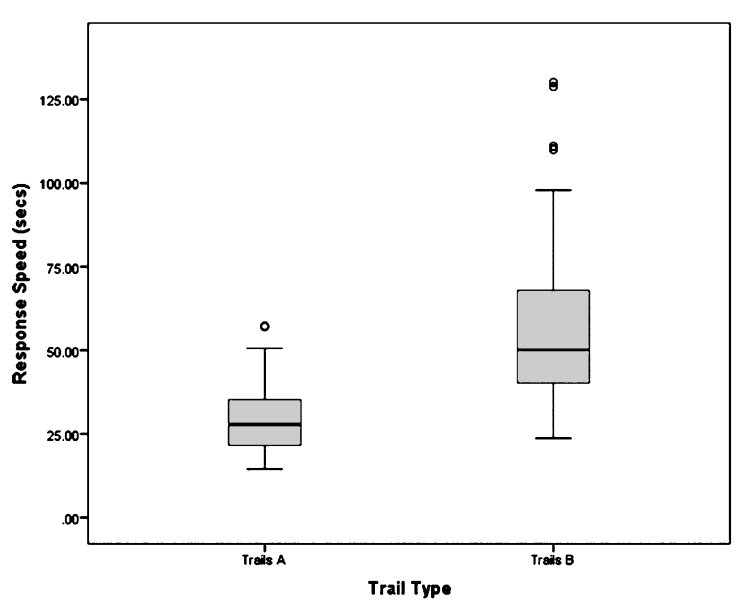

Fig. 1. Box plot of mean information processing speed (s) for Trails A and B performance in older adults.

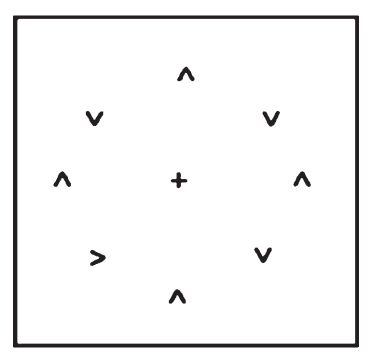

Fig. 2. Search stimulus.

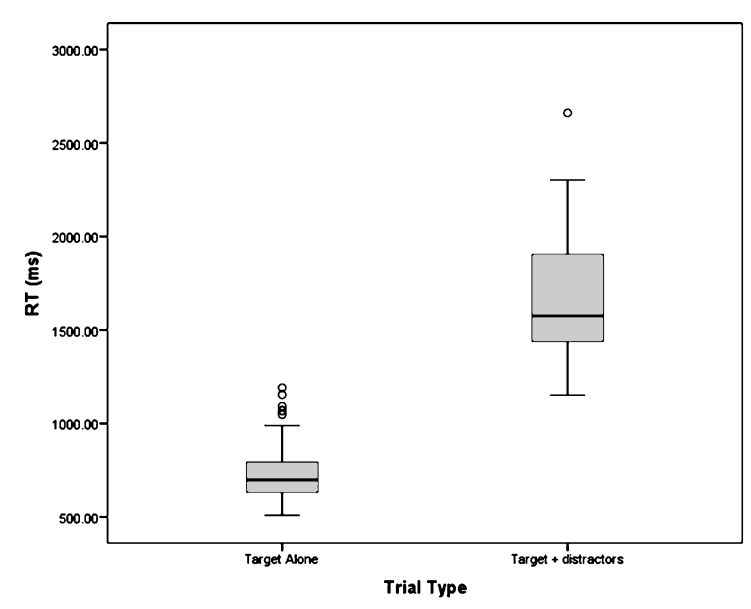

Fig. 3. Box plot of mean information processing speed (ms) for target alone and target plus distracters trials. 


\section{Visual search: information processing speed} and perceived task difficulty

The mean (sd) perceived task difficulty score for the visual search task was 3 (1.4) with a range from 1 to 6 . This was significantly negatively correlated with the target alone condition $(\mathrm{r}=-0.294, p=0.031)$, with slower actual information processing speed associated with less perceived task difficulty, but not for the target plus distracters conditions $(p>0.05)$. The number of errors was not significantly correlated with perceived task difficulty ( $p$ values $>0.05$ ).

\section{Subjective memory function and perceived task difficulty}

MFQ total score was not significantly correlated with perceived task difficulty for either the target alone or the target plus distracters condition ( $p$ values $>0.05$ ). Post hoc tests revealed that all the above results did not vary with respect to whether the participant was male or female.

\section{Educational level and information processing speed}

Further post hoc analysis revealed that although educational level was not significantly related to subjective memory performance it was significantly positively correlated with perceived test difficulty $(\mathrm{r}=0.440, p=0.01)$ and it was significantly negatively correlated with information processing speed for target plus distracter $(r=-0.398, p=0.003)$ condition, but not for the target alone condition $(p>0.05)$, i.e., a higher level of education is related to faster information processing speed when distractors are present.

\section{DISCUSSION}

The aim of these studies was to determine if a significant relationship exists between information processing speed, subjective memory function, and perceived task performance difficulty in older adults. In a difference of approach from some previous studies, we looked at people recruited from the community rather than from clinical settings, with normal general cognition and without significant depression or anxiety.

\section{Information processing speed and subjective memory}

Greater levels of overall subjective memory complaint (MFQ total score) were not significantly associated with slower information processing speed as measured by the TMT or Visual Search Task. For Trails A there was a significant negative correlation between information processing speed and the mnemonics subset of the MFQ and although this survived Bonferroni correction, the significance level of this effect was low $(p=0.042)$. For Trails B and both conditions of the visual search test, information processing speed was not significantly correlated with any of the MFQ subsets.

Given the close relationship between information processing speed and white and grey matter structure [6-12], the general absence of an association with perceived memory dysfunction in otherwise cognitively healthy, euthymic older adults suggests perceived memory dysfunction is less likely to be related to structural abnormality or possible neurodegenerative change. Similarly, given the association between Alzheimer's disease, vascular dementia and MCI and slowed information processing speed the lack of a relationship between perceived memory function and information processing speed may be indicative of a non-neurodegenerative basis for perceived impairment in individuals with 'normal' levels of general cognition (e.g., MoCA score within the normal range).

Note, however, that these ideas are speculative in nature given the absence of neuroimaging, a full range of objective and subjective measures of memory, cognitive and information processing speed performance, and longitudinal analysis examining the risk of developing MCI and/or dementia. The possibility that changes in brain structure and function and in memory may occur in the absence of changes in information processing speed must also be considered. Individuals also may perceive problems with memory but still perform at normal information processing speed as there may be, for some tasks, factors which influence memory and perceived memory but not information processing speed.

Nevertheless, if such findings were found to be robust after further research and development, the measurement of behavioral information processing speed might be of use in helping to determine for whom priority should be given with respect to further investigation and follow up of subjective memory complaints. For example, disproportionately slower 
responses (such as the outliers evident in our results, see Figs. 1 and 3) may be representative of detrimental change in underlying structure and function and thus a greater possibility of underlying neurodegeneration. Clinical follow-up and medical intervention with these patients could be prioritized, whereas others may benefit more from focus on psychological support, adjusting expectations regarding normal changes in cognitive performance, and providing metacognitive strategies to reduce the required effort for everyday tasks.

\section{Perception of task difficulty and subjective memory}

Greater subjective memory complaint was associated with a perception of greater task difficulty for both Trails A and Trails B of the TMT, especially for the more difficult Trails B test, with higher levels of subjective memory complaint related to greater perceived task difficulty. With respect to the TMT therefore, individuals who reported higher levels of perceived memory dysfunction (total MFQ score) are those who also reported the greater levels of task difficulty, despite actual (objectively measured) information processing speed not being associated with perceived memory function. To speculate, the relationship between perceived memory and perceived task difficulty in individuals with MOCA scores within the normal range in the absence of objective change in information processing speed suggests that perceived memory dysfunction may be related more to metacognitive factors than underlying structural change.

In contrast, for the visual search task, greater subjective memory complaint was not associated with a perception of greater task difficulty for both the target alone and the target plus distracter conditions. Individuals who reported higher levels of memory dysfunction did not report greater levels of task difficulty.

Such outcome variability indicates that the metacognition relationship between perceived memory and difficulty of task may not be generalizable to all tests, but mediated by factors such as difficulty, resource requirements and brain functions and areas recruited. However, different participants were used in the two studies and so the outcome effects may not be directly comparable. Nevertheless, our results raise the possibility that examining patterns of perceived memory function and the perception of task difficulty may help to determine whether perceived memory impairment (in the absence of objective change in memory function) is related to structural change or metacognition (which is more likely to be responsive to intervention and treatment than structural change), or indeed whether a much more complex relationship exists between metacognition, structural change and actual and perceived functional integrity.

\section{Information processing speed and perceived task difficulty}

For Trails A, perceived task difficulty was not significantly correlated with objectively measured information processing speed. In contrast, perceived task difficulty was positively correlated with information processing speed for Trails B, i.e., slower information processing speed was associated with greater perceived task difficulty. For the target plus distracters condition of the visual search task, perceived task difficulty was not significantly correlated with objectively measured information processing speed. For the target alone condition however, perceived task difficulty was significantly negatively correlated with information processing speed, i.e., slower information speed was associated with a lower level of perceived task difficulty. This pattern of results indicates that, irrespective of perceived memory function, the judgement of task difficulty is related to the nature of the task and is not always related to actual performance.

\section{Educational level}

For both Trails A and B, educational level was not significantly associated with information processing speed, subjective memory function or perceived task difficulty. For the visual search task, educational level was also not significantly related to subjective memory performance but it was significantly positively correlated with perceived test difficulty, i.e., a higher level of education was associated with higher levels of perceived task difficulty. Furthermore, educational level was significantly negatively correlated with information processing speed for the target plus distracter condition, i.e., a higher level of education was associated with faster information processing. In contrast, educational level was not significantly correlated with information processing speed for the target alone condition. Although educational level was not significantly related to perceived memory function, this pattern of results indicates the potential 
influence of education upon perceived task performance and actual task performance per se and a failure to take such factors into account in research may influence the interpretation of study outcome.

\section{Study limitations}

The participants in each study cohort were relatively young (mean age $65 \mathrm{y}$ ). However, this is the age group when subjective memory changes are typically reported and when pathological changes of neurodegenerative disease start to become more common. Future work, including neuroimaging, needs to explore the structural and functional relationships between information processing speed, actual and perceived memory function and metacognition factors in older age groups and in those seeking clinical diagnosis and with respect to mood and personality. Further research is also required to investigate why some individuals do not approach health care services about their perceived memory function. Some individuals may be anxious about a formal diagnosis, fearful that they may be developing dementia and unaware of other possible reversible causes of impairment or potential interventions to improve quality of life.

We tested participants on only one occasion and could not take into account the possibility that perceived memory may fluctuate, as it can be due to temporary conditions such as fatigue, or everyday stressful life events. Perceived memory was measured using only the MFQ. Related to this issue is the fact that we were not able to determine the accuracy of self-report on this measure. Furthermore, we did not include a battery of tests objectively measuring memory and cognition, while information processing speed was measured by only two tests, both of which had large executive function components. Nor did we measure separate specific components of behavioral information processing speed, such as motor function. The relationship between actual and perceived memory function, information processing speed and indeed metacognitive processes such as the perception of task difficulty, remains to be determined in relation to a variety of different tests, and ones that recruit different brain structure and functional domains and specific metacognitive factors. Finally, although it may not be possible to control medication, future studies should record and report medication in order to facilitate a greater understanding the generalizability of results to the population in general.

\section{ACKNOWLEDGMENTS}

The authors would like to thank all our participants, Dr. Jade Norris for participant recruitment, and BRACE-Alzheimer's Research for funding part of this study.

Authors' disclosures available online (http://j-alz. com/manuscript-disclosures/17-0599r1).

\section{REFERENCES}

[1] Salthouse TA (1996) The processing-speed theory of adult age differences in cognition. Psychol Rev 103, 403-428.

[2] Woods DL, Wyma JM, Yund EW, Herron TJ, Reed B (2015) Age-related slowing of response selection and production in a visual choice reaction time task. Front Hum Neurosci 9 , 193.

[3] Troyer AK, Vandermorris S, Murphy KJ (2016) Intraindividual variability in performance on associative memory tasks is elevated in amnestic mild cognitive impairment. Neuropsychologia 90, 110-116.

[4] Gorus E, De Raedt R, Lambert M, Lemper JC, Mets T (2008) Reaction times and performance variability in normal aging, mild cognitive impairment and Alzheimer's disease. J Geriatr Psychiatry Neurol 21, 204-218.

[5] Phillips M, Rogers P, Haworth J, Bayer A, Tales A (2013) Intra-individual reaction time variability in mild cognitive impairment and Alzheimer's disease: Gender, processing load and speed factors. PLoS One 8, e65712.

[6] Lu PH, Lee GL, Tishler TA, Meghpara M, Thompson PM, Bartzokis G (2013) Myelin breakdown mediates age-related slowing in cognitive processing speed in healthy elderly men. Brain Cogn 81, 131-138.

[7] Kerchner GA, Racine CA, Hale S, Wilheim R, Laluz V, Miller BL, Kramer JH (2012) Cognitive processing speed in older adults: Relationship with white matter integrity. PLoS One 7, e50425.

[8] Tamnes CK, Fjell AM, Westlye LT, Østby Y, Walhovd KB (2012) Becoming consistent: Developmental reductions in intraindividual variability in reaction time are related to white matter integrity. J Neurosci 32, 972-982.

[9] Nilsson J, Thomas AJ, O'Brien JT, Gallagher P (2014) White matter and cognitive decline in aging: A focus on processing speed and variability. J Int Neuropsychol Soc 20, 262-267.

[10] Hong Z, Ng KK, Sim SKY, Ngeow MY, Zheng H, Lo JC (2015) Differential age-dependent associations of gray matter volume and white matter integrity with processing speed in healthy older adults. Neuroimage 123, 42-50.

[11] Jacobs HIL, Leritz EC, Williams VJ, van Boxtel MPJ, van der Elst W, Jolles J (2013) Association between white matter microstructure, executive functions, and processing speed in older adults: The impact of vascular health. Hum Brain Mapp 34, 77-95.

[12] Yang Y, Bender AR, Raz N (2015) Age related differences in reaction time components and diffusion properties of normal-appearing white matter in healthy adults. Neuropsychologia 66, 246-258.

[13] Strauss E, Bielak AA, Bunce D, Hunter MA, Hultsch DF (2007) Within-person variability in response speed as an indicator of cognitive impairment in older adults. Neuropsychol Dev Cogn B Aging Neuropsychol Cogn 14, 608-630. 
[14] Fjell AM, Walhovd KB (2010) Structural brain changes in aging: Courses, causes and cognitive consequences. Rev Neurosci 21, 187-221.

[15] Lin F, Chen D-G, Vance D, Mapstone M (2013) Trajectories of combined laboratory- and real world-based speed of processing in community-dwelling older adults. J Gerontol Series B: Psychol Sci Soc Sci 68, 364-373.

[16] Batterham PJ, Bunce D, Mackinnon AJ, Christensen H (2014) Intra-individual reaction time variability and allcause mortality over 17 years: A community-based cohort study. Age Ageing 43, 84-90.

[17] Welmer AK, Rizzuto D, Qiu C, Caracciolo B, Laukka EJ (2014) Walking speed, processing speed, and dementia: A population-based longitudinal study. J Gerontol A Biol Sci Med Sci 69, 1503-1510.

[18] Anstey KJ, Dear K, Christensen H, Jorm AF (2005) Biomarkers, health, lifestyle, and demographic variables as correlates of reaction time performance in early, middle, and late adulthood. QJEP Section A: Hum Exp Psychol 58, 5-21.

[19] Eckert MA (2011) Slowing down: Age-related neurobiological predictors of processing speed. Front Neurosci $\mathbf{5}, 25$.

[20] Hagger-Johnson GE, Shickle DA, Roberts BA, Deary IJ (2012) Neuroticism combined with slower and more variable reaction time: Synergistic risk factors for 7-year cognitive decline in females. J Gerontol B Psychol Sci Soc Sci 67, 572-581.

[21] American Psychiatric Association (2013) Diagnostic and Statistical Manual of Mental Disorders: DSM-5. 5th ed. American Psychiatric Association, Washington, DC.

[22] Jessen F, Amariglio RE, van Boxtel M, Breteler M, Ceccaldi M, Chételat G, Dubois B, Dufouil C, Ellis KA, van der Flier WM, Glodzik L, van Harten AC, de Leon MJ, McHugh P, Mielke MM, Molinuevo JL, Mosconi L, Osorio RS, Perrotin A, Petersen RC, Rabin LA, Rami L, Reisberg B, Rentz DM, Sachdev PS, de la Sayette V, Saykin AJ, Scheltens P, Shulman MB, Slavin MJ, Sperling RA, Stewart R, Uspenskaya O, Vellas B, Visser PJ, Wagner M, Subjective Cognitive Decline Initiative (SCD-I) Working Group (2014) A conceptual framework for research on subjective cognitive decline in preclinical Alzheimer's disease. Alzheimers Dement 10, 844-852.

[23] Mark RE, Sitskoorn MM (2013) Are subjective cognitive complaints relevant in preclinical Alzheimer's disease? A review and guidelines for healthcare professionals. Rev Clin Gerontol 23, 61-74.

[24] Mitchell AJ, Beaumont H, Fergusen D, Yadegarfar M, Stubbs B (2014) Risk of dementia and mild cognitive impairment in older people with subjective memory complaints: Meta-analysis. Acta Psychiat Scand 130, 439-451.
[25] Mendonça MD, Alves L, Bugalho P (2015) From subjective cognitive complaints to dementia: Who is at risk? A systematic review. Am J Alzheimers Dis Other Demen 31, 105-114.

[26] Roehr S, Villringer A, Angermeyer MC, Luck T, RiedelHeller SG (2016) Outcomes of stable and unstable patterns of subjective cognitive decline - results from the Leipzig longitudinal study of the aged (LEILA75+). BMC Geriatrics 16, 180.

[27] João AA, Maroco J, Ginó S, Mendes T, de Mendonça A, Martins IP (2016) Education modifies the type of subjective memory complaints in older people. Int J Geriatr Psychiatry 31, 153-160.

[28] Barba GD, La Corte V, Dubois B (2015) For a cognitive model of subjective memory awareness. J Alzheimers Dis 48, S57-S61.

[29] Hill NL, Mogle J, Wion R, Munoz E, DePasquale N, Yevchak AM, Parisi JM (2016) Subjective cognitive impairment and affective symptoms: A systematic review. Gerontologist 56, e109-e127.

[30] Haworth J, Phillips M, Newson M, Rogers P, TorrensBurton A, Tales A (2016) Measuring information processing speed in mild cognitive impairment: Clinical versus research dichotomy. J Alzheimers Dis 51, 263-275.

[31] Fleming SM, Dolan RJ (2012) The neural basis of metacognitive ability. Philos Trans $R$ Soc Lond B Biol Sci 367, 1338-1349.

[32] Reitan RM (1958) Validity of the trail making test as an indicator of organic brain damage. Percept Mot Skills 8 , 271-276.

[33] Nasreddine ZS, Phillips NA, Bédirian V, Charbonneau S, Whitehead V, Collin I, Cummings JL, Chertkow H (2005) The Montreal Cognitive Assessment (MoCA): A brief screening tool for mild cognitive impairment. J Am Geriatr Soc 53, 695-699.

[34] Spitzer R, Kroenke K, Williams JB, Löwe B (2006) A brief measure for assessing generalized anxiety disorder: The GAD-7-7. Arch Int Med 166, 1092-1097.

[35] Löwe B, Decker O, Müller S, Brähler E, Schellberg D, Herzog W, Herzberg PY (2008) Validation and standardization of the Generalized Anxiety Disorder Screener (GAD-7-7) in the general population. Med Care 46, 266-274.

[36] Kroenke K, Spitzer RL, Williams JB (2001) The PHQ-9: Validity of a brief depression severity measure. J Gen Int Med 16, 606-613.

[37] Gilewski MJ, Zelinski EM, Schaie KW (1990) The Memory Functioning Questionnaire for assessment of memory complaints in adulthood and old age. Psychol Aging 5, 482-490. 